\title{
Electroweak Higgs Boson Plus Three Jet Production at Next-to-Leading-Order QCD
}

\author{
Francisco Campanario, ${ }^{1}$ Terrance M. Figy, ${ }^{2}$ Simon Plätzer, ${ }^{3}$ and Malin Sjödahl ${ }^{4}$ \\ ${ }^{1}$ Theory Division, IFIC, University of Valencia-CSIC, E-46100 Paterna, Valencia, Spain \\ ${ }^{2}$ School of Physics and Astronomy, University of Manchester, Manchester M13 9PL, United Kingdom \\ ${ }^{3}$ Theory Group, DESY, D-22607 Hamburg, Germany \\ ${ }^{4}$ Department of Astronomy and Theoretical Physics, Lund University, Sölvegatan 14A, 22362 Lund, Sweden
}

(Received 13 September 2013; published 18 November 2013)

\begin{abstract}
We calculate next-to-leading order (NLO) QCD corrections to electroweak Higgs boson plus three jet production. Both vector boson fusion (VBF) and Higgs-strahlung type contributions are included along with all interferences. The calculation is implemented within the MATCHBOX NLO framework of the HERWIG++ event generator.
\end{abstract}

DOI: 10.1103/PhysRevLett.111.211802

PACS numbers: $14.80 . \mathrm{Bn}, 12.38 . \mathrm{Bx}$

Introduction.-Higgs boson production via vector boson fusion (VBF) is an essential channel at the LHC for disentangling the Higgs boson's coupling to fermions and gauge bosons. The observation of two tagging jets is crucial to reduce the backgrounds. Furthermore, the possibility of identifying Higgs boson production via VBF is enhanced by being able to increase the signal to background ratio by vetoing additional soft radiation in the central region [1-7], since this suppresses important QCD backgrounds, including $H j j$ via gluon fusion.

To exploit the central jet veto (CJV) strategy for Higgs coupling measurements, the reduction factor caused by the CJV on the observable signal must be accurately known. The fraction of VBF Higgs events with at least one additional veto jet between the tagging jets provides the relevant information, i.e., we need to know the ratio of $H j j j$ production to the inclusive cross section of $H j j$ production via VBF. Gluon fusion next-to-leading order (NLO) QCD corrections for $H j j j$ within the heavy top effective theory approximation have been recently computed in [8]. A validation of the heavy top effective theory approximation at LO for this process can be found in [9].

At present, the NLO QCD corrections of $H j j j$ via VBF have been calculated [10] with several approximations and without the inclusion of five- and six-point one-loop Feynman diagrams (Fig. 1, second row) and the corresponding real emission cuts, which were estimated to contribute at the per mille level. However, some studies [5] suggest that the contribution of the missing pieces can be larger. Because of the importance of the process to Higgs measurements, a full calculation is necessary to ensure that integrated cross sections and kinematic distributions are not underestimated.

In this Letter, we present results for electroweak Higgs boson plus three jet production at NLO QCD. In the section "Calculational details," we present technical details of our computation. In the section "Numerical results," we present numerical results and the impact of the NLO QCD corrections on various differential distributions.
Finally, we summarize our findings in the Conclusions section.

Calculational details.-For the calculation of the LO matrix elements for Higgs plus two (we have performed the $\mathrm{HjJ}$ calculation in parallel within the same framework), three, and four jets, we have used the built-in spinor helicity library of the MATCHBOX module [11] to build up the full amplitude from hadronic currents. For the Born-virtual interference, the helicity amplitude methods described in [12] have been employed. Both methods resulted in two different implementations of the tree level amplitudes which have been validated against each other, including color correlated matrix elements. The LO implementation has further been cross checked against SHERPA $[13,14]$ and HAWK $[15,16]$. The dipole subtraction terms [17] have been generated automatically by the MATCHBOX module [11], which is also used for a diagram-based multichannel phase space sampler. For the virtual corrections, we have used in-house routines, extending the techniques developed in [18]. The amplitudes have been cross checked against GOSAM [19]. A representative set of contributing topologies are depicted in Fig. 1.
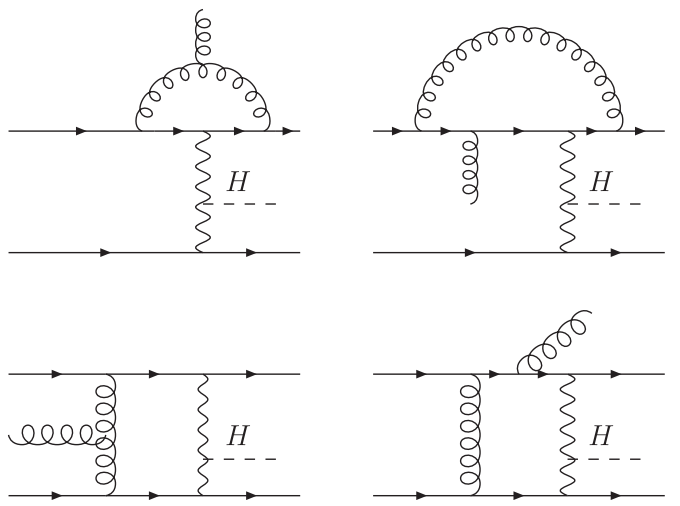

FIG. 1. Representative examples of diagrams of $H j j j$ production via vector boson fusion. 
For electroweak propagators, we have introduced finite width effects following [20], using complex gauge boson masses and a derived complex value of the sine of the weak mixing angle. We used the ONELOOP library [21] which supports complex masses to calculate the scalar integrals. For the reduction of the tensor coefficients up to four-point functions, we apply the Passarino-Veltman approach [22], and for the numerical evaluation of the five and six point coefficients, we use the DennerDittmaier scheme [23], following the layout and notation of [18].

To ensure the numerical stability of our code, we have implemented a test based on Ward identities [18]. These Ward identities are applied to each phase space point and diagram, at the expense of a small additional computing time and using a cache system. If the identities are not fulfilled, the amplitudes of gauge related topologies are set to zero. The occurrence of these instabilities are at the permille level, and therefore well under control. This method was also successfully applied in other processes with two to four kinematics $[24,25]$. In the present work, it is applied for the first time to a process which involves loop propagators with complex masses.

The color algebra has been performed using COLORFULL and cross-checked using COLORMATH [27]. Within the same framework, we have implemented the corresponding calculation of electroweak $H j j$ production and performed cross checks against HAWK $[15,16]$.

Numerical results. - In our calculation, we choose $m_{Z}=$ $91.188 \mathrm{GeV}, \quad m_{W}=80.419002 \mathrm{GeV}, \quad m_{H}=125 \mathrm{GeV}$, and $G_{F}=1.16637 \times 10^{-5} \mathrm{GeV}^{-2}$ as electroweak input parameters and derive the weak mixing angle $\sin \theta_{W}$ and $\alpha_{\mathrm{QED}}$ from standard model tree level relations. All fermion masses (except the top quark) are set to zero and effects from generation mixing are neglected. The widths are calculated to be $\Gamma_{W}=2.0476 \mathrm{GeV}$ and $\Gamma_{Z}=2.4414 \mathrm{GeV}$. We use the CT10 [28] parton distribution functions with $\alpha_{s}\left(M_{Z}\right)=0.118$ at NLO, and the CTEQ6L1 set [29] with $\alpha_{s}\left(M_{Z}\right)=0.130$ at LO. We use the five-flavor scheme and the center-of-mass energy is fixed to $\sqrt{s}=14 \mathrm{TeV}$.

To study the impact of the QCD corrections, we use minimal inclusive cuts. We cluster jets with the anti- $k_{T}$ algorithm [30] using FASTJET [31] with $D=0.4$, $E$-scheme recombination and require at least three jets with transverse momentum $p_{T, j} \geq 20 \mathrm{GeV}$ and rapidity $\left|y_{j}\right| \leq 4.5$. Jets are ordered in decreasing transverse momenta.

In Fig. 2, we show the LO and NLO total cross sections for inclusive cuts for different values of the factorization and renormalization scale varied around the central scale, $\mu$ for two scale choices, $M_{W} / 2$, and the scalar sum of the jet transverse momenta, $\mu_{R}=\mu_{F}=\mu=H_{T} / 2$ with $H_{T}=\sum_{j} p_{T, j}$. In general, we see a somewhat increased cross section and-as expected-decreased scale dependence in the NLO results. We also note that the central

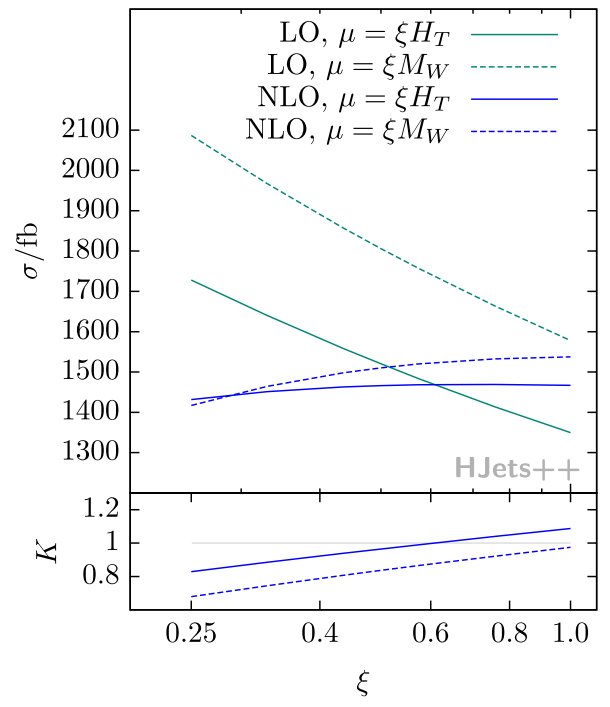

FIG. 2 (color online). The $H j j j$ inclusive total cross section (in fb) at LO (cyan) and at NLO (blue) for the scale choices, $\mu=\xi M_{W}$ (dashed line) and $\mu=\xi H_{T}$ (solid line). We also show the $K$ factor, $K=\sigma_{\mathrm{NLO}} / \sigma_{\mathrm{LO}}$ for $\mu=\xi M_{W}$ (dashed line) and $\mu=\xi H_{T}$ (solid line).

values for the various scale choices are closer to each other at NLO. The uncertainties obtained by varying the central value by a factor 2 up and down are around $30 \%$ $(24 \%)$ at LO and $2 \%(9 \%)$ at NLO using $H_{T} / 2\left(M_{W} / 2\right)$ as the scale choice. At $\mu=H_{T} / 2$, we obtained $\sigma_{\mathrm{LO}}=$ $1520(8)_{-171}^{+208} \mathrm{fb} \sigma_{\mathrm{NLO}}=1466(17)_{-35}^{+1} \mathrm{fb}$. Studying differential distributions, we find that these generally vary less using the scalar transverse momentum sum choice, used from now on.

In the following, we show some of the differential distributions which characterize the typical vector boson fusion selection cuts and central jet veto strategies as well as the $p_{T}$ spectrum of the Higgs boson.

For the leading jets (defined to be the two jets with highest transverse momenta $p_{T, 1}$ and $p_{T, 2}$ ), we show the rapidity difference in Fig. 3. Generally, we find small differences in shape compared to the $\mathrm{LO}$ results.

In Fig. 4, the differential distribution for the $p_{T}$ of the Higgs boson is shown. The NLO corrections are moderate over the whole spectrum and the scale uncertainties are clearly smaller.

In Fig. 5, the differential distribution of the third jet, the vetoed jet for a CJV analysis, is presented. Here we find large differences in the high energy tail of the transverse momentum distribution. Such high energy jets are significantly enhanced at NLO.

We show in Fig. 6 the normalized centralized rapidity distribution of the third jet with respect to the tagging jets, $z_{3}^{*}=\left[y_{3}-(1 / 2)\left(y_{1}+y_{2}\right)\right] /\left(y_{1}-y_{2}\right)$. This variable beautifully displays the VBF nature present in the process. One clearly sees how the third jet tends to accompany one of the leading jets appearing at $1 / 2$ and $-1 / 2$, 


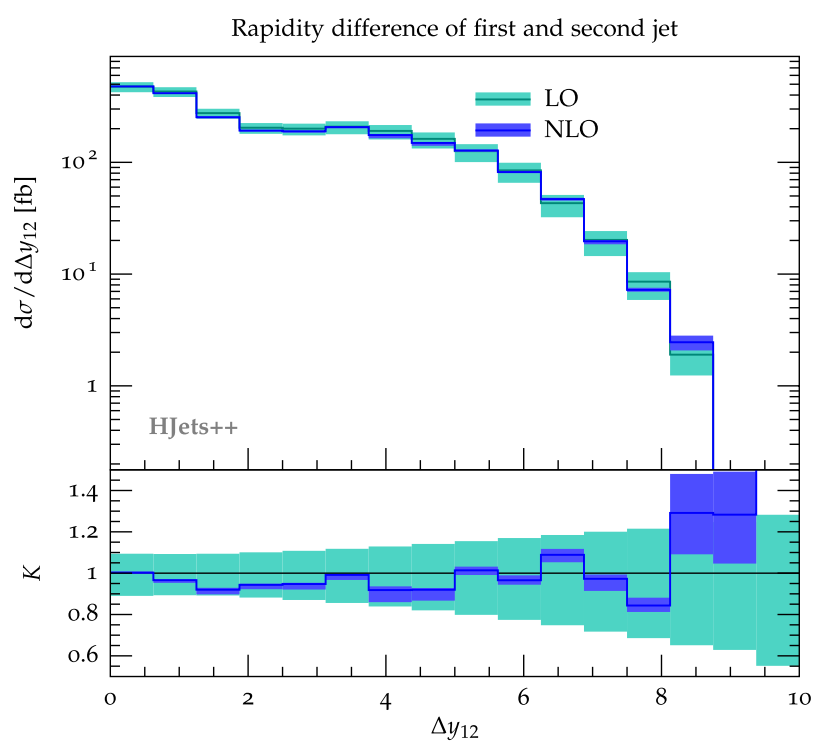

FIG. 3 (color online). Differential cross section and $K$ factor for the rapidity separation of the leading two jets. Cuts are described in the text. The bands correspond to varying $\mu_{F}=\mu_{R}$ by factors $1 / 2$ and 2 around the central value $H_{T} / 2$.

respectively. This effect is expected to be yet more pronounced when VBF cuts are applied, and should be contrasted with the gluon fusion production mechanism where radiation in the midrapidity region will be much more common due to the $t$-channel color flow of the process [5,7-9]. Note also that the ordering of the leading jets in transverse momenta generates an asymmetric

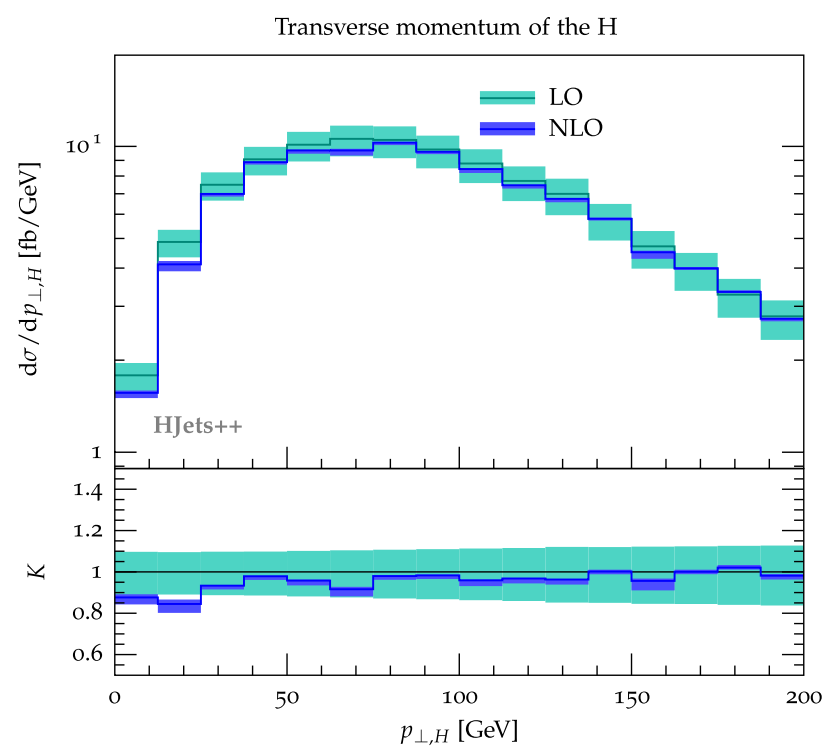

FIG. 4 (color online). Differential cross section and $K$ factor for the $p_{T}$ of the Higgs boson. Cuts are described in the text. The bands correspond to varying $\mu_{F}=\mu_{R}$ by factors $1 / 2$ and 2 around the central value $H_{T} / 2$.

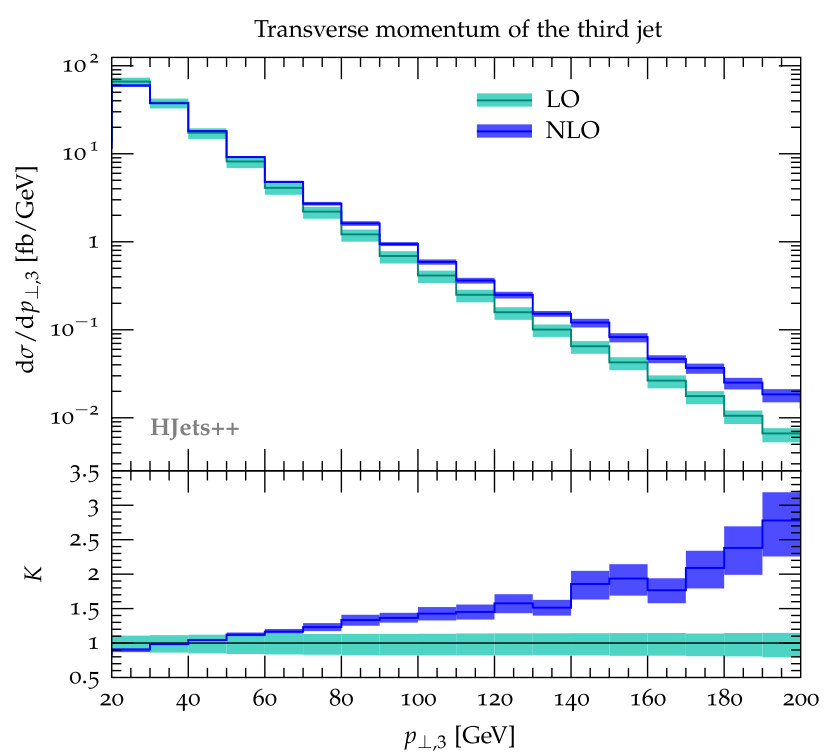

FIG. 5 (color online). Differential cross section and $K$ factor for the $p_{T}$ of the third hardest jet. Cuts are described in the text. The bands correspond to varying $\mu_{F}=\mu_{R}$ by factors $1 / 2$ and 2 around the central value $H_{T} / 2$.

radiation pattern. There is more radiation around $z_{3}^{*}=1 / 2$ corresponding to emission of the third jet along the first leading jet, $y_{3}=y_{1}$. Additionally, we remark, however, that studying the rapidity distribution of the third jet alone does not display the same characteristic pattern; here the peaks accompanying the forward jets in the VBF region are completely smeared out (not shown here).

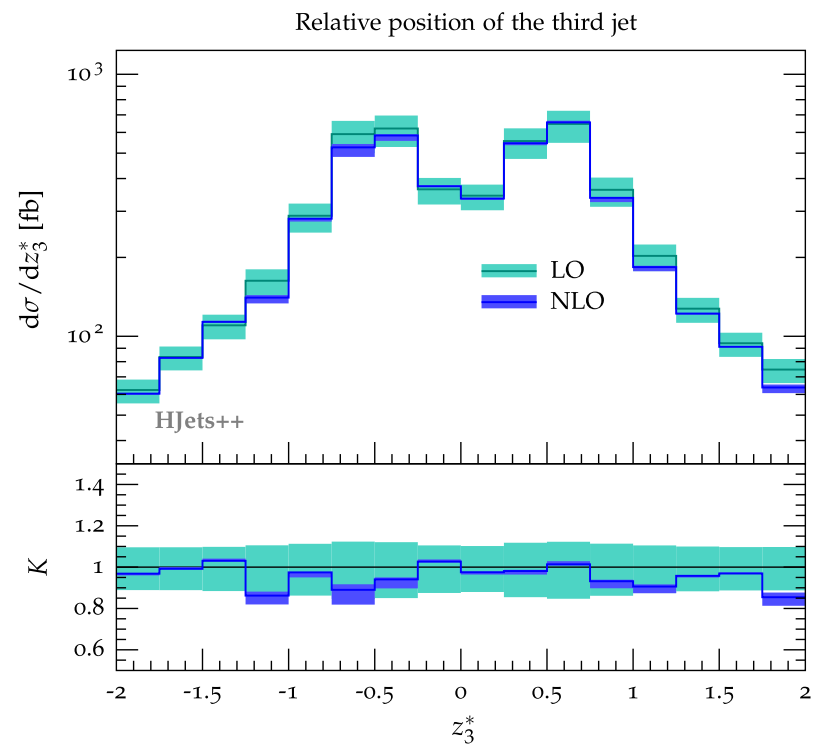

FIG. 6 (color online). Differential cross section and $K$ factor for the normalized centralized rapidity distribution of the third jet with respect to the tagging jets. Cuts are described in the text. The bands correspond to varying $\mu_{F}=\mu_{R}$ by factors $1 / 2$ and 2 around the central value $H_{T} / 2$. 
Conclusions. - In this Letter, complete results at NLO in perturbative QCD for electroweak Higgs boson production in association with three jets have been presented. The NLO corrections to the total inclusive cross section are moderate for the inclusive cuts and a central scale of $H_{T} / 2$ considered here, but can be more significant for a scale choice of $M_{W} / 2$. However, they exhibit a nontrivial phase space dependence reflected in nonconstant differential $K$ factors, particularly for jet $p_{T}$ spectra. The scale uncertainty significantly decreases from around $30 \%(24 \%)$ at LO down to about $2 \%(9 \%)$ at NLO using $H_{T} / 2\left(M_{W} / 2\right)$.

Despite our choice of inclusive cuts which allow for the possibility that jets are radiated in the central region, Higgs boson plus three jet production prefers VBF kinematics as seen in the $z^{*}$ differential distribution. A detailed discussion of VBF cuts and jet vetoes will be given elsewhere, including an assessment of the region of validity of the VBF approximation. Results including the effect of parton showering are also anticipated. The code is available upon request and will be publicly available in the near future.

We are grateful to Ken Arnold for contributions at an early stage of this project and to Mike Seymour and Jeff Forshaw for valuable discussions on the subject. F. C. is funded by a Marie Curie fellowship (PIEF-GA-2011298960) and partially by MINECO (FPA2011-23596) and by LHCPhenonet (PITN-GA-2010-264564). T.F. would like to thank the North American Foundation for The University of Manchester and George Rigg for their financial support. S. P. has been supported in part by the Helmholtz Alliance "Physics at the Terascale" and M.S. was supported by the Swedish Research Council, Contract No. 621-2010-3326.

[1] V. D. Barger, R. Phillips, and D. Zeppenfeld, Phys. Lett. B 346, 106 (1995).

[2] D. L. Rainwater, D. Zeppenfeld, and K. Hagiwara, Phys. Rev. D 59, 014037 (1998).

[3] D. L. Rainwater and D. Zeppenfeld, Phys. Rev. D 60, 113004 (1999).

[4] V. Del Duca, A. Frizzo, and F. Maltoni, J. High Energy Phys. 05 (2004) 064.

[5] J. R. Forshaw and M. Sjodahl, J. High Energy Phys. 09 (2007) 119.

[6] J. R. Andersen, V. Del Duca, and C. D. White, J. High Energy Phys. 02 (2009) 015.
[7] B. E. Cox, J. R. Forshaw, and A. D. Pilkington, Phys. Lett. B 696, 87 (2011).

[8] G. Cullen, H. van Deurzen, N. Greiner, G. Luisoni, P. Mastrolia, E. Mirabella, G. Ossola, T. Peraro, and F. Tramontano, Phys. Rev. Lett. 111, 131801 (2013).

[9] F. Campanario and M. Kubocz, Phys. Rev. D 88, 054021 (2013).

[10] T. Figy, V. Hankele, and D. Zeppenfeld, J. High Energy Phys. 02 (2008) 076.

[11] S. Platzer and S. Gieseke, Eur. Phys. J. C 72, 2187 (2012).

[12] K. Hagiwara and D. Zeppenfeld, Nucl. Phys. B313, 560 (1989).

[13] T. Gleisberg, S Hoeche, F Krauss, A Schaelicke, S Schumann, and J Winter, J. High Energy Phys. 02 (2004) 056

[14] T. Gleisberg, S Höche, F Krauss, M Schönherr, S Schumann, F Siegert, and J Winter, J. High Energy Phys. 02 (2009) 007.

[15] M. Ciccolini, A. Denner, and S. Dittmaier, Phys. Rev. Lett. 99, 161803 (2007).

[16] M. Ciccolini, A. Denner, and S. Dittmaier, Phys. Rev. D 77, 013002 (2008).

[17] S. Catani and M.H. Seymour, Nucl. Phys. B485, 291 (1997).

[18] F. Campanario, J. High Energy Phys. 10 (2011) 070.

[19] G. Cullen, N. Greiner, G. Heinrich, G. Luisoni, P. Mastrolia, G. Ossola, T. Reiter, and F. Tramontano, Eur. Phys. J. C 72, 1889 (2012).

[20] A. Denner and S. Dittmaier, Nucl. Phys. B, Proc. Suppl. 160, 22 (2006).

[21] A. van Hameren, Comput. Phys. Commun. 182, 2427 (2011).

[22] G. Passarino and M. Veltman, Nucl. Phys. B160, 151 (1979).

[23] A. Denner and S. Dittmaier, Nucl. Phys. B734, 62 (2006).

[24] F. Campanario, C. Englert, M. Rauch, and D. Zeppenfeld, Phys. Lett. B 704, 515 (2011).

[25] F. Campanario, M. Kerner, L. D. Ninh, and D. Zeppenfeld, Phys. Rev. Lett. 111, 052003 (2013).

[26] M. Sjodahl http://colorfull.hepforge.org.

[27] M. Sjodahl, Eur. Phys. J. C 73, 2310 (2013).

[28] H.-L. Lai, M. Guzzi, J. Huston, Z. Li, P. M. Nadolsky, J. Pumplin, and C.-P. Yuan, Phys. Rev. D 82, 074024 (2010).

[29] J. Pumplin, D. R. Stump, J. Huston, H.-L. Lai, P. Nadolsky, and W.-K. Tung, J. High Energy Phys. 07 (2002) 012

[30] M. Cacciari, G. P. Salam, and G. Soyez, J. High Energy Phys. 04 (2008) 063.

[31] M. Cacciari, G. P. Salam, and G. Soyez, Eur. Phys. J. C 72, 1896 (2012). 